\title{
Detection of epithelial to mesenchymal transition in airways of a bleomycin induced pulmonary fibrosis model derived from an $\alpha$-smooth muscle actin-Cre transgenic mouse
} Zhuang $\mathrm{Wu}^{\dagger 1}$, Leilei Yang ${ }^{\dagger 2}$, Lin Cai ${ }^{1}$, Min Zhang1, Xuan Cheng ${ }^{2}$, Xiao Yang*2 and Jun $\mathrm{Xu}^{* 1}$

Address: ${ }^{1}$ Guangzhou Institute of Respiratory Disease, First Affiliated Hospital of Guangzhou Medical College, Guangzhou, 510120, P. R. China and ${ }^{2}$ Genetic Laboratory of Development and Diseases, Institute of Biotechnology, 20 Fengtai Eastern Street, Beijing, 100071, P.R.China

Email: Zhuang Wu - wuzhuang@126.com; Leilei Yang - qqneversaydie@yahoo.com.cn; Lin Cai - lin_cai@126.com; Min Zhang - zhangmincc@sohu.com; Xuan Cheng - xuan_cheng@sohu.com; Xiao Yang* - yangx@nic.bmi.ac.cn; Jun Xu* - xufeili@vip.163.com

* Corresponding authors †Equal contributors

Published: 07 January 2007

Respiratory Research 2007, 8:1 doi:10.1 I86/1465-992I-8-1
Received: 02 September 2006

Accepted: 07 January 2007

This article is available from: http://www.biomedcentral.com/I465-992I/8/I

(c) 2007 Wu et al; licensee BioMed Central Ltd.

This is an Open Access article distributed under the terms of the Creative Commons Attribution License (http://creativecommons.org/licenses/by/2.0), which permits unrestricted use, distribution, and reproduction in any medium, provided the original work is properly cited.

\begin{abstract}
Background: Epithelial to mesenchymal transition (EMT) in alveolar epithelial cells (AECs) has been widely observed in patients suffering interstitial pulmonary fibrosis. In vitro studies have also demonstrated that AECs could convert into myofibroblasts following exposure to TGF- $\beta$ I. In this study, we examined whether EMT occurs in bleomycin (BLM) induced pulmonary fibrosis, and the involvement of bronchial epithelial cells (BECs) in the EMT. Using an $\alpha$-smooth muscle actin-Cre transgenic mouse ( $\alpha$-SMA-Cre/R26R) strain, we labelled myofibroblasts in vivo. We also performed a phenotypic analysis of human BEC lines during TGF- $\beta$ I stimulation in vitro.

Methods: We generated the $\alpha$-SMA-Cre mouse strain by pronuclear microinjection with a Cre recombinase cDNA driven by the mouse $\alpha$-smooth muscle actin ( $\alpha$-SMA) promoter. $\alpha$-SMA-Cre mice were crossed with the Cre-dependent LacZ expressing strain R26R to produce the double transgenic strain $\alpha$-SMA-Cre/R26R. $\beta$ galactosidase ( $\beta$ gal) staining, $\alpha$-SMA and smooth muscle myosin heavy chains immunostaining were carried out simultaneously to confirm the specificity of expression of the transgenic reporter within smooth muscle cells (SMCs) under physiological conditions. BLM-induced peribronchial fibrosis in $\alpha$-SMA-Cre/R26R mice was examined by pulmonary $\beta$ gal staining and $\alpha$-SMA immunofluorescence staining. To confirm in vivo observations of BECs undergoing EMT, we stimulated human BEC line $16 \mathrm{HBE}$ with TGF- $\beta$ I and examined the localization of the myofibroblast markers $\alpha$-SMA and F-actin, and the epithelial marker E-cadherin by immunofluorescence.

Results: $\beta$ gal staining in organs of healthy $\alpha-S M A-C r e / R 26 R$ mice corresponded with the distribution of SMCs, as confirmed by $\alpha-S M A$ and SM-MHC immunostaining. BLM-treated mice showed significantly enhanced $\beta$ gal staining in subepithelial areas in bronchi, terminal bronchioles and walls of pulmonary vessels. Some AECs in certain peribronchial areas or even a small subset of BECs were also positively stained, as confirmed by $\alpha$-SMA immunostaining. In vitro, addition of TGF- $\beta$ I to I6HBE cells could also stimulate the expression of $\alpha$-SMA and Factin, while E-cadherin was decreased, consistent with an EMT.

Conclusion: We observed airway EMT in BLM-induced peribronchial fibrosis mice. BECs, like AECs, have the capacity to undergo EMT and to contribute to mesenchymal expansion in pulmonary fibrosis.
\end{abstract}




\section{Background}

Myofibroblast cells, an intermediate cell type between fibroblasts and smooth muscle cells (SMCs), have been suggested to play an important role in the development of interstitial pulmonary fibrosis (IPF), which produces excessive amounts of extracellular matrix (ECM), leading to formation of fibroblastic foci [1-3]. However, much is still unknown regarding the origin of myofibroblasts and the process resulting in devastating airway aggravation. Previously, it was suggested that peribronchiolar and perivascular fibroblasts transdifferentiate into myofibroblasts following exposure to profibrotic mediators such as TGF- $\beta 1$ [4]. Alternatively, airway SMCs might dedifferentiate into myofibroblasts, but this possibility has been ruled out by several studies suggesting that ultrastructural features and ECM expression profiles of myofibroblasts are more similar to fibroblasts than to SMCs $[1,5]$. Recently, fibrocytes originating in the bone marrow have been proposed to be recruited into the lung after bleomycin (BLM) administration and to act as myofibroblast progenitors [6]. More recently, alveolar epithelial cells (AECs) have been shown to undergo epithelial to mesenchymal transition (EMT) to produce myofibroblasts in IPF patients and following TGF- $\beta 1$ treatment in vitro [7-9]. Moreover, EMT in AECs has been demonstrated in a mouse pulmonary fibrosis model [10]. The BLM induced peribronchial fibrosis mouse model largely recapitulates histological features of human pulmonary fibrosis [11], and thus provides a convenient and powerful in vivo tool that has been the most widely used animal model to study the pathogenetic mechanisms of pulmonary fibrosis. However, the common BLM-induced pulmonary fibrotic model is derived from wild mouse and thus is unsuitable for tracking the origin of active myofibroblasts in the development of pulmonary fibrosis, due to their great "plasticity" and tendency to switch to other phenotypes [12].

In the present study, we employed the Cre/LoxP recombinase system, using the $\alpha$-smooth muscle actin ( $\alpha$-SMA) promoter to drive Cre-dependent recombination in presumptive myofibroblast cells as well as SMCs. We then generated an $\alpha$-SMA-Cre/R26R transgenic mouse strain that allows permanent $\beta$-galactosidase ( $\beta$ gal) labeling in airway SMCs and the other structural cells undergoing transdifferentiation into myofibroblasts. Since the recombination is achieved by Cre-dependent removal of the transcriptional stop sequence between the two LoxP sites upstream of the lacZ gene in R26R mice, lacZ expression will permanently label Cre-expressing cells $[13,14]$. As expected, our transgenic mouse model accurately labeled the distribution of SMCs in various organs under physiological conditions; cumulatively recorded the activation of myofibroblasts in the lung under BLM induced fibrotic conditions and revealed EMT occurring in AECs and even in BECs. Moreover, to verify the occurrence of EMT in BECs in vitro, we treated the human BEC cell line 16HBE with TGF- $\beta 1$, which was also capable of inducing EMT.

\section{Methods \\ Reagents}

For histological immunofluorescent staining, anti- $\alpha$-SMA monoclonal antibody (mAb) was purchased from Sigma (reactive with human and mouse $\alpha$-SMA, Cat A2547); anti-bovine smooth muscle myosin heavy chains (SM$\mathrm{MHC}$ ) polyclonal antibody (pAb) was kindly provided by Professor Mary Anne (NIH/NHLBI, US); rabbit antihuman E-cadherin pAb was purchased from Santa Cruz Biotechnology (Cat sc-7870), rabbit anti-mouse/human E-cadherin pAb was purchased from Boster Company (Cat BA0475). GAPDH mAb was purchased from Chemicon (Cat CB1001). Secondary antibodies of goat anti-rabbit pAb conjugated with FITC and goat anti-mouse pAb conjugated with TRITC were purchased from Bethyl(Cat A120-201F) and Open Biosystems (Cat SAB1428), respectively. Goat anti-mouse pAb conjugated to HRP was purchased from Santa Cruz Biotechnology (Cat sc-2005). Bleomycin (BLM) used for establishing the peribronchial fibrosis model was purchased from Nipponkayaku (Tokyo, Japan). Primers were synthesized in Sangon (Shanghai, China). All chemicals for $\beta$ gal staining were purchased from Jingmei Company (Shenzhen, China).

\section{Generation of the $\tilde{\alpha}-S M A-C r e / R 26 R$ transgenic mouse strain}

The Cre recombinase CDNA was PCR amplified from the pMCI-13Cre plasmid (a gift from Professor F. Costantini, Department of Genetics, Columbia University, NY, USA) using the following primers: forward 5'GAAGATCTATGCCCAAGAAGAAGAGGAAGGTGTCCAATTTACTGAC-3' and reverse 5'-CGGAATTCTGAACAAACGACCCAAC-3'. The PCR product was then sub-cloned into the BamHI-EcoRI site of the VSMP8 plasmid (a gift of Professor Art Strauch, Dorothy M. Davis Heart and Lung Research Institute, Columbus, OH, USA) which contains the mouse $\alpha \mathrm{SMA}$ promoter fragment 1070 +2582, including the first exon and part of first intron (GenBank: U63129 and M57409). The $\alpha$-SMA promoter-Cre fragment was released from the construct using Sphl and EcoRI for transgenic microinjection (Fig. 1). Transgenic $\alpha$-SMA-Cre mice were produced by pronuclear injection of the recombinant DNA fragment into fertilized F2 eggs of CBA mice using standard microinjection techniques. Offspring from an $\alpha$-SMA-Cre-carrying transgenic founder mouse were selected and crossed to the Cre dependent conditional reporter strain R26R+/+ (Rosa26, Soriano P)[15]. 


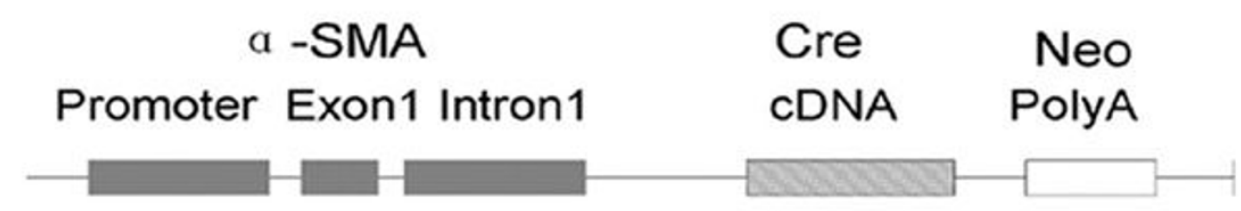

\section{R26R}

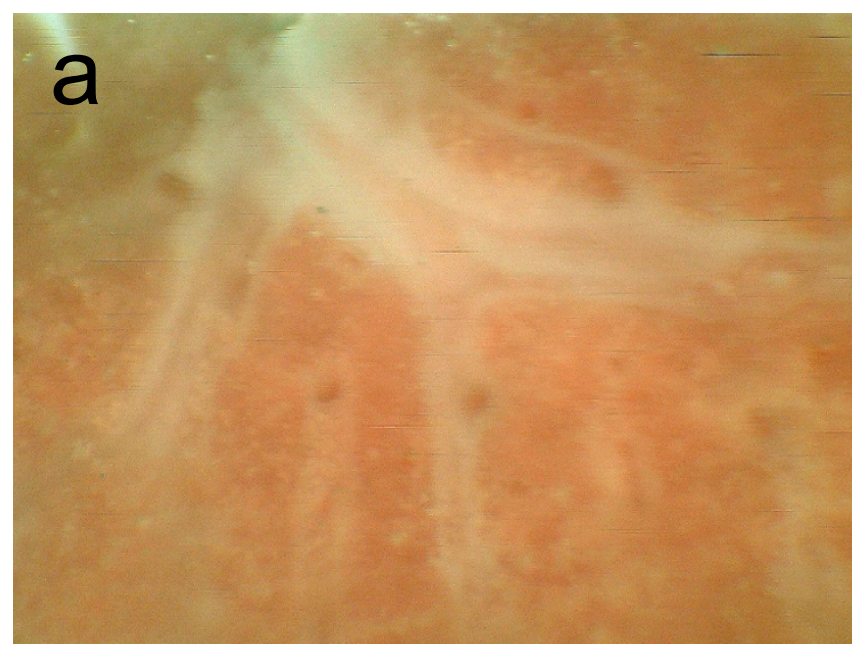

SMA-Cre/R26R

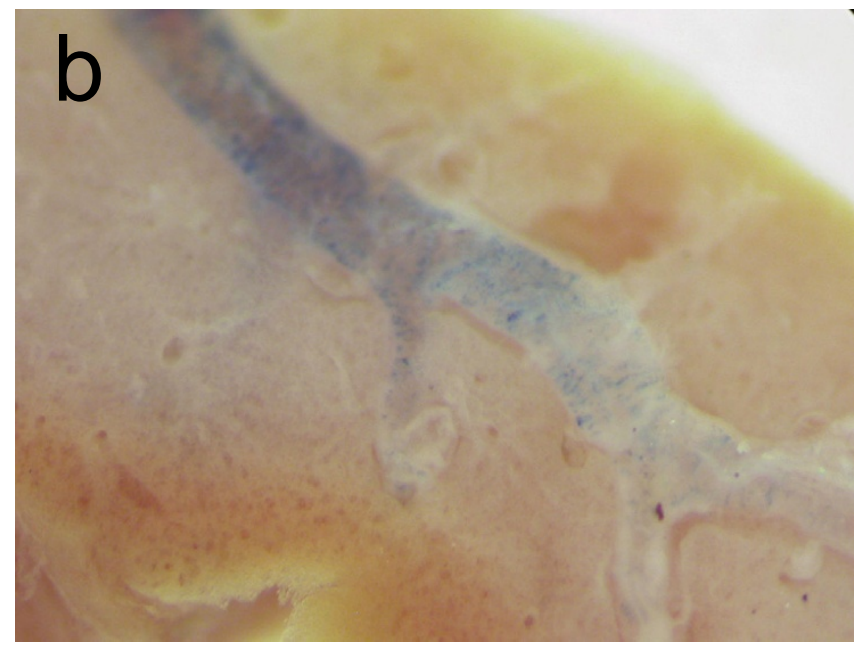

\section{Figure I}

Transgene construction and $\beta$ gal staining of lung lobes. A. Transgene fragment for microinjection. The Cre $c D N A$ and a Neo polyadenylylation signal were placed under the control of the mouse $\alpha$-SMA promoter $(-1070$ to +2582 , including the first exon and part of the first intron). B. Comparison of $\beta$ gal staining in the bronchi of R26R and $\alpha-S M A-C r e / R 26 R$ mice. Positive $\beta$ gal staining (blue color) is observed in the bronchi of $\alpha-S M A-C r e / R 26 R$ mice (a, 20× magnification), but not in R26R mice (b, 20× magnification).

\section{Generation of the BLM-induced pulmonary fibrosis mouse model}

5-6 wk old SMA-Cre/R26R mice were endotracheally injected with $80 \mu \mathrm{l} \mathrm{BLM} \mathrm{(3} \mathrm{mg/kg} \mathrm{in} \mathrm{PBS)} \mathrm{or} \mathrm{with} 80 \mu \mathrm{l}$ PBS ( $n=4$ for each group). These mice were sacrificed 20 days later for western blot analysis, $\beta$ gal and immunofluorescent staining.

\section{Tissue $\beta$-galactosidase ( $\beta$ gal) staining}

Organs were dissected from BLM or PBS treated transgenic mice and subjected to $\beta$ gal staining. Briefly, organs were fixed in $0.1 \mathrm{M}$ PBS, pH7.3 containing $0.25 \%$ glutaraldehyde, $2 \mathrm{mM} \mathrm{MgCl} 2,5 \mathrm{mM}$ EGTA at $4{ }^{\circ} \mathrm{C}$ for $1-2 \mathrm{hrs}$. Left lung lobes were perfused with $1 \mathrm{ml}$ fixing solution by endotracheal injection and right lobes were ligated and removed. Tissues were then incubated in wash buffer $(0.1$

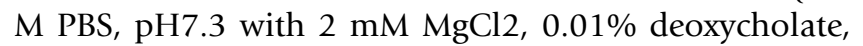
$0.02 \%$ NP-40) 3 times for $30 \mathrm{~min}$ each, and then in staining buffer (0.1 M PBS, pH7.3 with $1 \mathrm{mg} / \mathrm{ml} \beta g a l, 2 \mathrm{mM}$ $\mathrm{MgCl} 2,0.01 \%$ deoxycholate, $5 \mathrm{mM} \mathrm{K3Fe}(\mathrm{CN}) 6,6 \mathrm{mM}$ $\mathrm{K} 4 \mathrm{Fe}(\mathrm{CN}) 6,0.02 \% \mathrm{NP}-40)$ at $37^{\circ} \mathrm{C}$ overnight. Following staining, wholemount tissues were observed under XTL3400 Zoom Stereo Microscope (CANY, Shanghai, China) or processed by dehydrating, wax embedding, sectioning at $8 \mu \mathrm{m}$ intervals and counterstaining with Carmine Alum. Microscopic analyses were performed with a Leica DM LB2 microscope equipped with a digital camera.

\section{Lung histology and immunohistochemistry}

After sacrificing $\alpha$-SMA-Cre/R26R mice, right lung lobes (upper and middle lobes) were dissected and fixed in formalin and processed by conventional histological procedures. After sectioning at $4 \mu \mathrm{m}$ intervals, sections were dewaxed, rehydrated, blocked with $10 \%$ goat serum for 60 min at room temperature and immunofluorescently stained with $\alpha$-SMA, SM-MHC or E-cadherin. Sections were incubated with anti- $\alpha$-SMA mAb (1:400), antibovine SM-MHC pAb (1:400) or co-incubated with E-cadherin pAb $(1: 100)$ overnight at $4^{\circ} \mathrm{C}$ and subsequently incubated with goat anti-mouse IgG-TRITC (1:800) pAb or goat anti-rabbit IgG-FITC (1:400) pAb for 1 hour. DAPI was used to stain nuclei (500 ng/ml in 95\% ethanol) for 
$20 \mathrm{sec}$, and coverslips were mounted with $80 \%$ glycerol. Slides were examined using a Leica DC 500-fluorescence microscope equipped with a digital camera.

Alternatively, lung sections were processed for Masson's trichrome staining to detect collagen and elastin. The staining was carried out using Masson trichrome staining Kit (Maxim-Bio, Fuzhou, China) according to the manufacturer's instruction.

\section{Western Blot}

$\alpha$-SMA protein levels in lungs were evaluated by western blot as previously described [16]. After cytoplasmic protein extraction from the lower lobe of right lung of PBS or BLM-injected mice, protein was quantified using a BCA assay kit (Pierce, USA) and $20 \mu \mathrm{g}$ was used for SDS-PAGE electrophoresis. Following electrophoretic transfer, membranes were incubated with anti- $\alpha$-SMA mAb $(1: 1000)$ in TBS/T buffer at $4{ }^{\circ} \mathrm{C}$ overnight. Membranes were incubated with anti-mouse IgG secondary antibody conjugated to HRP (1:1000), followed by exposure to ECL chemiluminescent substrate (Amersham, UK) and digital scanning in Image station 2000 (Kodak, US). Following $\alpha$-SMA blotting, films were placed in stripping buffer (50 $\mathrm{mM}$ DTT, $50 \mathrm{mM}$ Tris. HCI, $2 \%$ SDS) at $50^{\circ} \mathrm{C}$ for $30 \mathrm{~min}-$ utes, washed 5 times, reblocked and reprobed with GAPDH mAb (1:800) and HRP conjugated secondary antibody. Then the membranes went through chemiluminescence as discribed above to detect GAPDH protein in the same film. $\alpha$-SMA protein levels were measured by densitometry, and expressed relative to GAPDH. Duplicate samples were analyzed for each mouse.

\section{Cell culture and immunofluorescent staining}

The human bronchial epithelial cell line 16HBE-14o (16HBE), a generous gift from Professor S. Holgate (Southampton University, UK) was routinely maintained in growth medium consisting of MEM (Life Technologies, USA) and 10\% FCS (Shijiqing Co, China). Cells were seeded into sterile round coverslips placed inside 12-well plates. On reaching $70 \%$ confluence, medium was changed to FCS-free MEM, and rhTGF- $\beta 1$ (R\&D company, US) was added to a subset of wells to a final concentration of $10 \mu \mathrm{g} / \mathrm{L}$. 72 hours later, all wells were washed twice with cold PBS and a subset of wells were fixed with cold methanol:acetone $(1: 1)$ at $-20^{\circ} \mathrm{C}$ for 10 min. Coverslips were removed from the wells and placed on glass slides, blocked with $10 \%$ goat serum for $60 \mathrm{~min}$. Cells on coverslips were incubated with anti $\alpha$-SMA mAb $(1: 400)$ or rabbit anti human E-cadherin (1:50) overnight at $4{ }^{\circ} \mathrm{C}$ and subsequently incubated with goat anti-mouse IgG-TRITC $(1: 800)$ or goat anti-rabbit IgG-FITC $(1: 400)$ for 1 hour. Another subset of wells were fixed with PFA at RT for 20 min and treated with $0.1 \%$ TritonX-100 for $5 \mathrm{~min}$. Coverslips were removed from wells, placed on slides, blocked with $10 \%$ goat serum for $30 \mathrm{~min}$ and incubated with 100 $\mu \mathrm{l}$ Alex 594 phalloidin (1:500) for $20 \mathrm{~min}$ at RT. DAPI was used to stain nuclei $(500 \mathrm{ng} / \mathrm{ml}$ in $95 \%$ ethanol) for 20 sec, and coverslips were mounted with $80 \%$ glycerol. Slides were examined using a Leica DC 500-fluorescence microscope equipped with a digital camera.

\section{Results \\ Generation of $\alpha$-SMA-Cre/R26R transgenic mice}

To permanently label myofibroblasts, we firstly generated transgenic mice bearing an $\alpha$-SMA promoter driven Cre. Ten pseudopregnant mice were implanted oviductally with fertilized eggs injected with the construct, yielding 19 offspring, 4 of which were identified to carry the randomly integrated transgene. Two founder mice were selected and used to produce inbred strains. We then crossed an $\alpha$-SMA-Cre transgenic strain to reporter strain $\mathrm{R} 26 \mathrm{R}+/+$ whereby Cre-specific recombination at the ROSA26 locus allows expression of $\beta$-galactosidase in smooth muscle cells and myofibroblasts.

\section{Bgal staining corresponds with distribution of the smooth muscles of the $\alpha$-SMA-Cre R26R strain}

$\beta$ gal staining was performed on offspring of the $\alpha$-SMACre/R26R and the R26R mice respectively. Positive Bgal staining was observed in the trachea of the $\alpha$-SMA-Cre/ R26R strain (Fig. 1b), but not in that of the R26R mouse (Fig. 1a) under anatomy microscopy, confirming that the $\beta$ gal staining resulted from $\alpha$-SMA-driven Cre-mediated recombination.

As expected, $\beta$ gal staining was highly restricted to SMCs in smooth muscle-rich organs isolated from the $\alpha$-SMA-Cre/ R26R mice (data not shown). In pulmonary arteries and veins, $\beta$ gal staining was consistent with the natural distribution of smooth muscle tissue at these sites (Fig. 2a, b). In the intrapulmonary bronchus (Fig. $2 \mathrm{~d}, \mathrm{~g}$ ), the staining was precisely localized to the muscularis layer of the airway, which paralleled the immunofluorescent staining pattern of $\alpha$-SMA (Fig. 2e, h) and SM-MHC (Fig. 2f, i). Neither the terminal bronchioles, which lack SMC (Fig. 2c), nor the bronchial epithelia (Fig. 2a,b,c,d,g) showed positive $\beta$ gal staining.

\section{A great enhancement of $\beta$ gal staining in the lungs of the double transgenic mice after Bleomycin treatment}

The double transgenic mouse strain described above provides a simple means to follow expression of $\alpha$-SMA, and thus the regulation of myofibroblast development during pulmonary fibrosis. We next endotracheally administrated BLM in the transgenic mice for induction of lung injury and fibrosis. As shown in figure $3 \mathrm{~A} \sim \mathrm{b}$, in the wholemount lung preparations from BLM-treated $\alpha$-SMA-Cre/ R26R transgenic mice, $\beta$ gal staining is easily observed. In contrast, except for the helium area, the staining is not 


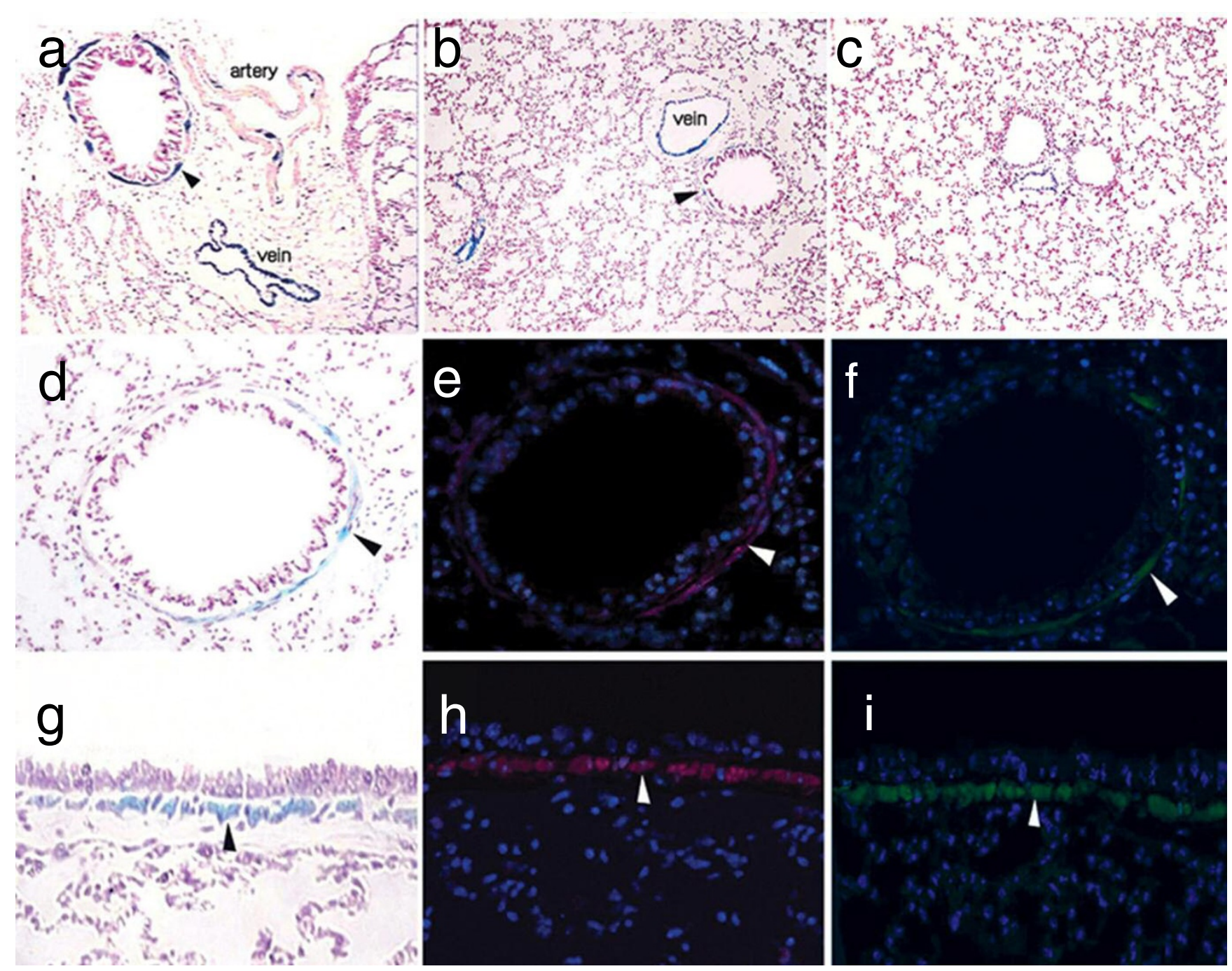

\section{Figure 2}

$\beta$ gal and immunofluorescent staining in lung tissues of $\alpha-S M A-C r e / R 26 R$ mice. In $\beta$ gal stained sections (a, b, c, d, g), intrapulmonary veins were homogeneously stained $(a, b)$ and pulmonary arteries were heterogeneously stained (a). In the main bronchus of pulmonary hilum, unstained ciliated epithelia were surrounded by a $\beta$ gal stained muscular layer (a, arrowhead), $\beta$ gal staining was not detected in terminal bronchioles, although small veins were positively stained (c). The thin layer of $\beta$ gal staining was observed in the sub-epithelial areas of small and medium bronchi, respectively (arrowheads in b, $d, g$ ). The $\beta g a l$ stained areas of bronchus $(d, g)$ paralleled the staining pattern for $\alpha$-SMA (TRITC-labeled, arrowheads in e, h) and SM-MHC (FITC-labeled, arrowheads in $\mathrm{f}, \mathrm{i}$ ) (a-c, 100x, d-i, 400x magnification).

observed in preparations from PBS-treated transgenic mice (Fig. 3A a).

Histological observations reveal a significantly increased number of $\beta$ gal staining- positive cells located at subepithelial areas of bronchioles, terminal bronchioles (Fig. $3 \mathrm{~A} \sim \mathrm{d}, \mathrm{f})$ and tunica media of pulmonary vessels, particularly pulmonary veins (Fig. 3A f, h arrowheads) in BLM treated mice. At these sites, distribution of collagen is visualized by Masson's trichrome staining, showing increased collagen deposition around the walls of small veins and terminal respiratory bronchioles and in certain parenchymal areas (Fig. 3A j arrowheads). In contrast, there is only minimal $\beta$ gal staining (Fig. $3 A \sim c, e, g$ ) and Masson trichrome staining (Fig. 3A i) in the lung of control mice.

Correspondingly, western blot analysis revealed an overall increase in lung $\alpha$-SMA protein in the BLM treated transgenic mice, compared with the control mice (Fig. 3B) 

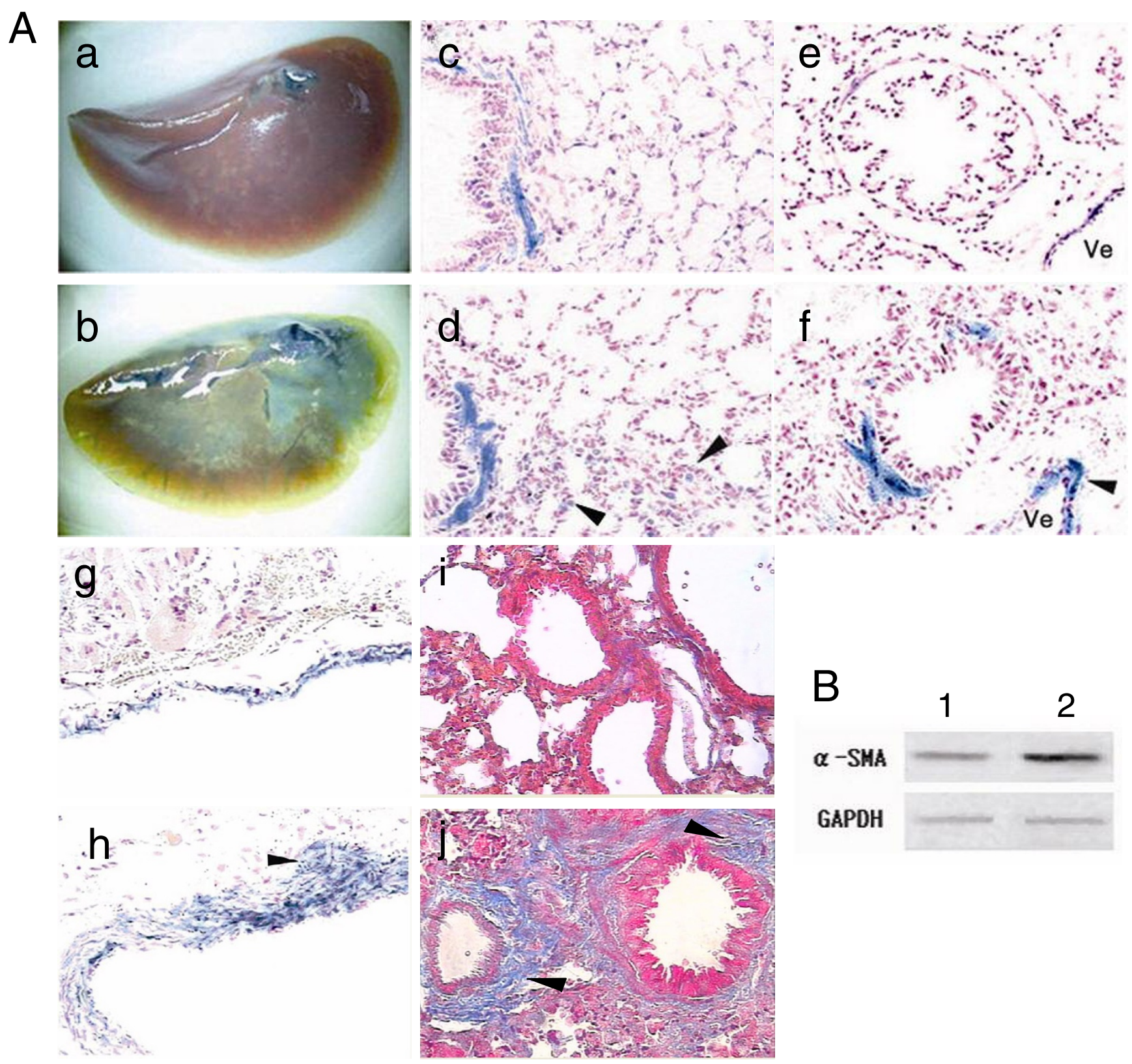

Figure 3

Effects of BLM on lung $\alpha$-SMA protein levels, ECM deposition and $\beta$ gal staining. Panel A: $\beta$ gal and Masson's trichrome-staining in sections of lung tissue shows $\beta$ gal staining to wholemount left lung lobes of the PBS-treated (a) and the BLM-treated mice (b) (a, b I0x magnification). In moderate bronchi, thickened bronchial wall with homogeneous $\beta$ gal stained fusiform cells was observed in the BLM-treated lungs (d), and not in the PBS-treated mouse (c), Arrowheads indicate that a few cells in alveolar wall were positively stained $(\mathrm{d})$. In pulmonary bronchioles and vessels, BLM treated lung demonstrated enhanced $\beta g$ al expression ( $f, h)$, compared with that of PBS treated lung (e, g). Bgal stained venous wall was thickened (arrowhead in $f$ ) and the positively stained cells infiltrated outwards (arrowhead in $h$ ). In the Masson's trichrome-stained lung sections $(i, j)$, extensive collagen staining (Blue color) was seen in BLM treated lung (arrowheads in j), but not in the control with PBS (i). (c-j 400x magnification). Panel B: Western blot analysis of protein extracts from lower right lobes of the BLM treated mice (2) and control mice (I). 


\section{Detection of EMT in bronchial epithelial cells of the $\alpha$ - SMA-Cre/R26R mice during BLM-induced pulmonary fibrosis}

As shown in Fig. 4, we also detected a few $\beta$ gal positive cells, with basal or columnar epithelial cell morphology, existing in epithelia lining the bronchioles (Fig. 4b, c arrowheads) and air-sacs (Fig. 3And arrowheads), in the BLM-treated transgenic mice. This was not observed in the control mice (Fig. 3A c,e; Fig. 4a). Using double immunofluorescent staining, with antibodies against $\alpha$-SMA and E-cadherin, we further demonstrated that certain cells located at bronchiolar epithelium of BLM treated mice were simultaneously stained with these epithelial and mesenchymal markers (Fig. 4g,h,i arrowheads), indicating their undergoing of EMT. No common-staining was found in control mice (Fig. 4d,e,f). We have also found similar results from lung tissues after BLM treatment at day $7,14,20$ and 28.

\section{In vitro phenotype analysis of I6HBE following exposure of TGF- $\beta$ I}

In vitro immunofluorescent staining of $16 \mathrm{HBE}$ cells (human bronchial epithelial cell line) demonstrates that exposure to TGF- $\beta 1$ results in an apparent reduction of Ecadherin staining, an epithelial marker, concomitant with its redistribution from intercellular junction areas into the cytoplasm (Fig. 5a, b). In contrast, the mesenchymal marker F-actin, whose expression was detectable only at the cellular margin before the exposure, shows an increased level in the epithelial cells where it is diffusely distributed throughout the cytoplasm after stimulation with TGF- $\beta 1$ (Fig. 5c, d). Meanwhile, positive $\alpha$-SMA immunofluorescent staining, which was undetectable prior exposure to TGF- $\beta 1$, appeared in the cytoplasm in a small number of the $16 \mathrm{HBE}$ cells (Fig. 5e, f).

\section{Discussion}

Using the Cre/Loxp system, we generated a transgenic mouse strain that expressed lacZ specifically in SMCs and myofibroblasts containing tissues. The $\beta$ gal expression pattern in the $\alpha$-SMA-Cre/R26R transgenic model closely resembled the expression of endogenous $\alpha$-SMA in the airways, and that in the gastrointestinal channel, vessels and genitourinary tract under normal physiological conditions. These data suggest that the SMP8 promoter region of the $\alpha$-SMA gene, including the first exon and part of the first intron $(-1070$ to +2582 of the mouse $\alpha$-SMA promoter), is sufficient to recapitulate endogenous $\alpha$-SMA expression patterns, in concordance with previous studies [17-19].

Smooth muscle-targeted Cre recombinase mice that have previously been generated by others for study of diseases, including SM22-CreER and SMMHC-Cre strains in which Cre is driven by the promoter of SM22 gene or SM-MHC gene, respectively. Feil and colleagues have generated the SM22-CreER transgenic mice to the effect that the expression of the transgene is confined to smooth muscle cells for studying vascular and gastrointestinal diseases [20]. However, gene knockout studies suggest that SM22 is not required for vascular and visceral SMC homeostatic functions in the developing mouse [21], and there are no data demonstrating that SM22 expression signifies myofibroblast activation. With regards to the SMMHC-Cre strain that has also been used to study vascular development and diseases [22], it has been documented that SM-MHC is seldom expressed in non-SMC cells such as myofibroblasts $[23,24]$. In contrast, our $\alpha$-SMA-Cre/R26R strain appears to be sensitive to myofibroblast activation after BLM exposure, as Cre-mediated recombination is controlled by the promoter of the gene encoding $\alpha$-SMA, a marker of myofibroblast transition.

Additionally, for the reason that in vivo recombination in Cre/Loxp system is irreversible, $\beta$ gal staining in the lung of our transgenic strain could reflect past and present myofibroblast transition events post BLM treatment. This may assist discovery of the cellular source of the active myofibroblasts in the development of pulmonary fibrosis. In the chronic progression of fibrosis, multiple cycles of injury and repair may occur repeatedly with a broad time period and range of sites. Activation of the $\alpha$-SMA promoter may be a transient event and limited to a subgroup of cells at a given time point [25]. So the $\alpha$-SMA-Cre mouse strain is likely to be highly relevant for studies of fibrotic diseases and activation of myofibroblasts, and trace the source of myofibroblasts.

In the BLM-treated $\alpha$-SMA-Cre/R26R mice, we observed a number of $\beta$ gal staining positive cells emerging in the subepithelial areas of bronchioles and terminal bronchioles and in the ectoblast of vessels, concomitant with extensive Masson Trichrome-stained extracellular matrix. In comparison, in the PBS-treated $\alpha$-SMA-Cre/R26R mice, $\beta$ gal positive cells were seldom seen, suggesting that not only can the reporter mice demonstrate the inherent distribution of pulmonary SMCs under physiologic condition, but also have the capability to sensitively record the trail of myofibroblast transition in the lung of the mice following pathologic stimulation. We demonstrate herein that increased $\beta$ gal expression in the lungs of the BLM-treated mice is mainly to be due to the appearance of myofibroblasts in the subepithelial areas of bronchiole and terminal bronchiole. Previous studies had shown that airway BLM administration does not result in remarkable morphological changes in the SMC layer [26].

There have been prior suggestions that EMT occurs in the lung during fibrogenesis, but these suggestions derive largely from studies of transformed cells or primary AECs 


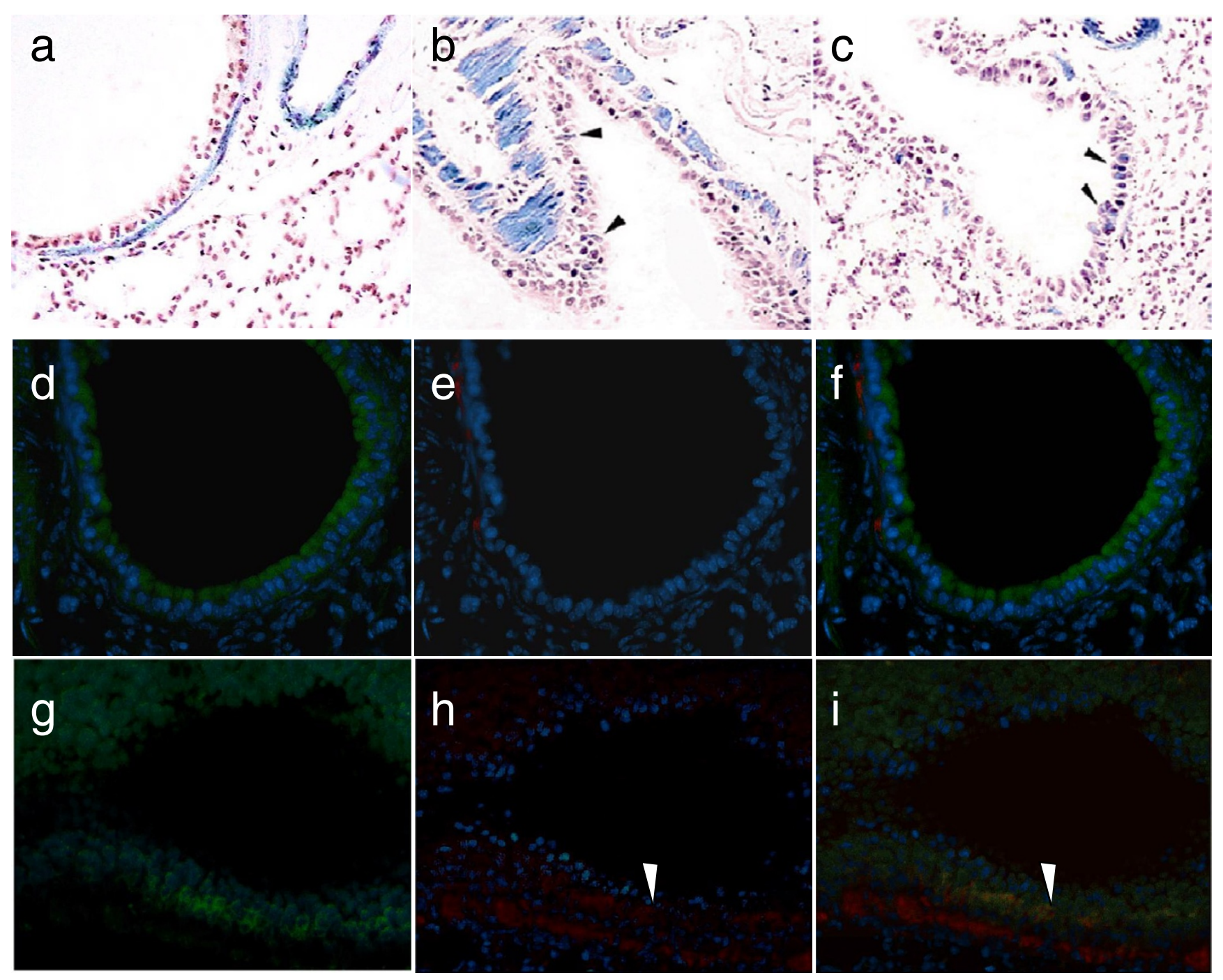

Figure 4

$\beta$ gal and $\alpha$ SMA positively stained bronchial epithelial cells in the $\alpha$-SMA-Cre R26R mice treated with BLM. $\beta$ gal stained lung sections of $\alpha$-SMA-Cre/R26R mice without and with BLM treatment (a-c). The section from BLM treated mice showed a few $\beta$ gal stained bronchial epithelial cells (arrowheads in b and c), but not from PBS treated mice (a). Double immunofluorescent staining for $\alpha-S M A$ and E-Cadherin was performed on the sections from PBS (d-f) or BLM (g-i) treated mice. $d$, g: FITC-labeled E-cadherin; e, h: TRITC labeled $\alpha$-SMA. Positive double immunofluorescent staining $(g, h, i)$ was observed in the bronchial epithelial cells lining the bronchioles of the BLM-treated lung where $\beta$ gal staining was detected as above, but not in the control (d, e, f). The images of (d) and (e), or (g) and (h) were merged into (f) and (i). The red fluorescence (h arrowhead) indicated the positive $\alpha$-SMA staining and yellow fluorescent staining ( $i$ arrowhead) indicated that the epithelial cells positively co-stained with $\alpha$-SMA and E-Cadherin. (all images are 400x magnification).

cultured on plastic, the in vivo significance of which is unclear $[7,9]$. It has recently been reported from IPF lung biopsies that epithelial cells had acquired mesenchymal features, raising the possibility of EMT during fibrogenesis [8]. More recently, Kim and colleagues developed a transgenic mouse reporter strain in which lung epithelial cells were genetically altered to permanently express $\beta g a l$, and their fates are followed in an established model of pulmonary fibrosis induced by intranasal Adeno-TGF- $\beta 1$. They showed that $\beta$ gal-positive cells expressing mesenchymal markers accumulated within 3 weeks of in vivo TGF- $\beta 1$ expression, demonstrating that EMT occurs in vivo in an animal model [10]. As shown at figure 3, we also observed the occurrence of EMT in parenchymal alveloar areas following BLM stimulation in our $\alpha$-SMA-Cre/R26R reporter mice where a few $\beta$ gal-positive cells located in alveolar wall demonstrated that the cells were undergoing EMT. Alternatively, the ßgal-stained epithelial cells may simply 

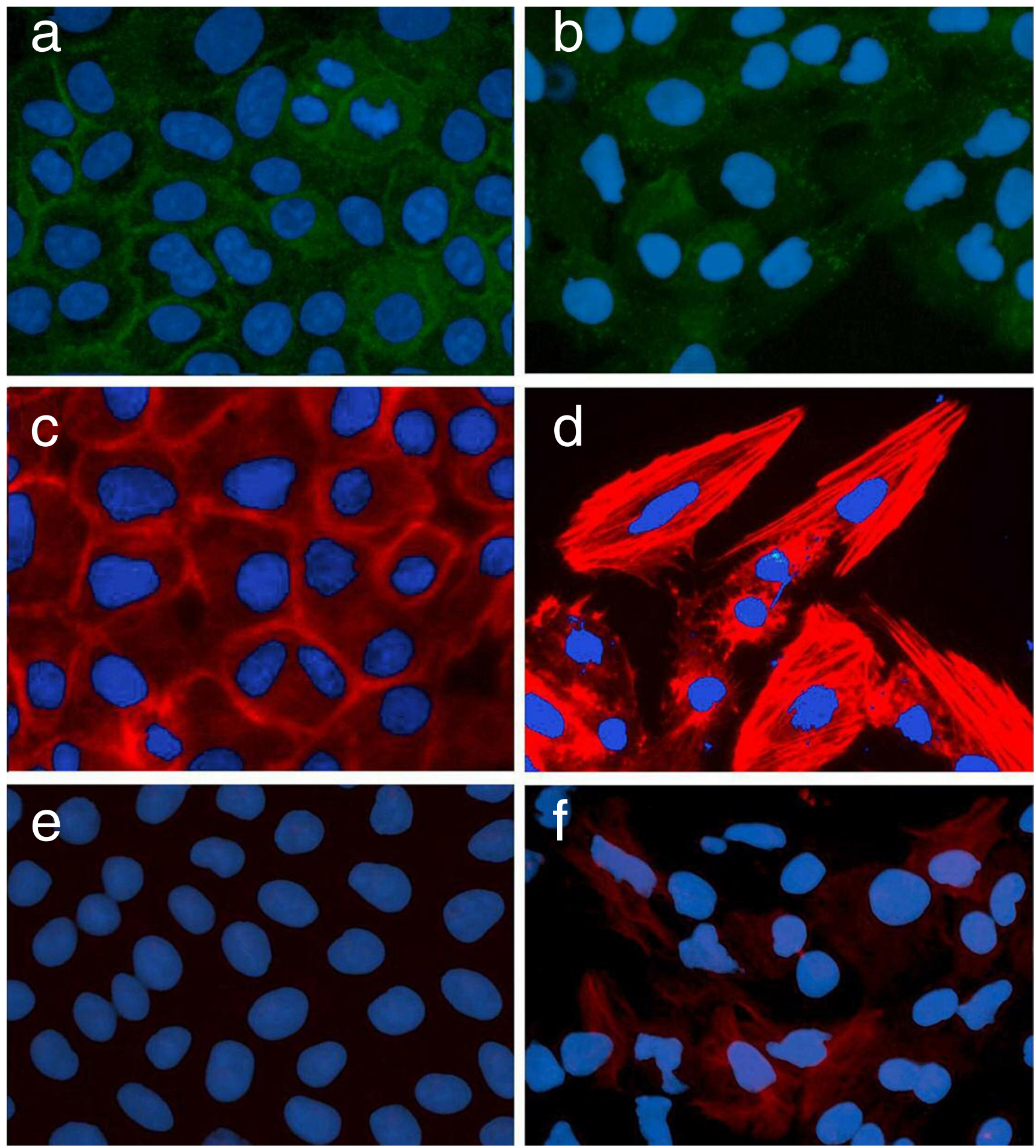

Figure 5

Phenotypic analysis of the human bronchial epithelial cell line (I6HBE) following exposure to TGF- $\beta$ I. Immunofluorescent staining for E-cadherin $(\mathrm{a}, \mathrm{b})$ showed that exposure to TGF- $\beta \mathrm{I}$ (b) resulted in an apparent reduction and redistribution of E-cadherin from intercellular junction areas into cytoplasm, compared to control (a). Mesenchymal marker F-actin, was faintly stained at the cell margin in the control (c), whereas the staining was substantially enhanced and abundantly located throughout cytoplasm after TGF- $\beta$ I stimulation (d). Immunofluorescent staining for (-SMA was not detected in the cells under basal conditions (e), but was observable in a few cells after TGF- $\beta$ I exposure (f). 
demonstrate transcriptional activation of $\alpha$-SMA gene in these cells.

Additionally, ßgal was stained positively in a few basal epithelial cells and columnar epithelial cells lining the bronchiole during bleomycin-induced lung fibrosis in the reporter mice. The immunofluorescent co-staining of the both E-cadherin and $\alpha$-SMA confirmed further that the BECs were undergoing EMT. When we focused on the BEC cell line 16HBE in vitro, we found that exposure to TGF$\beta 1$ led to a remarkable myofibroblast cell-like phenotype, marked by expression of $\alpha$-SMA and F-actin and the reduction of the epithelial-specific junction localization of E-cadherin. Taken together, these observations suggest that BECs might also be capable of undergoing EMT and thereby provide another cellular source for the parenchymal aggregation of myofibroblasts during fibrosis.

As mentioned above, however, the present histological observations in the reporter mice do not support the rationale that EMT exerts a critical influence on the progression of pulmonary fibrosis, because EMT indicated by $\beta$ gal staining and $\alpha$-SMA immunostaining was rarely detected in BECs and AECs of BLM-treated mice. For the predominant activation of sub-epithelial myofibroblasts in the development of BLM-treated lung fibrosis, fibroblasts or other sources of progenitors may play more essential roles which contribute to the pool of expanded myofibroblasts after lung injury. To what extent does EMT contribute to the aggravation of fibrosis, whether similar EMT in BECs occur in IPF patients are all interesting questions for future studies.

Additionally, the $\alpha$-SMA-Cre single transgenic strain bearing the $\alpha$-SMA driven Cre is sufficiently sensitive to test the function of a candidate gene in SMCs or myofibroblasts on the development of pulmonary fibrosis. Tissuespecific gene knockout or knock-in can be accomplished via crossing the $\alpha$-SMA-Cre mouse to a strain containing a loxP site flanked sequence of interest.

\section{Conclusion}

In conclusion, we have developed a double transgenic reporter mouse strain to map the natural distribution of $\alpha$-SMA-expressing cells in vivo under basal physiological condition. Moreover, lung cells that do not express $\alpha$-SMA under normal conditions may permanently express $\beta$ gal via $\alpha$-SMA activation in response to pathologic stimulation, thus allowing tracking of the cellular source of myofibroblasts and to definitively test whether EMT occurs in vivo.

\section{Competing interests}

The author(s) declare that they have no competing interests.

\section{Authors' contributions}

ZW carried out the transgene construction, transgenic screening and breeding, histological works and drafted the manuscript. LLY carried out the microinjections and transgenic screening and breeding. LC participated in the histological work and mice screening and breeding. $\mathrm{MZ}$ participated in the in vitro immunostaining. XC participated in the microinjections. $\mathrm{XY}$ guided the microinjection and animal breeding. JX design the study, technical support the research, revise the manuscript and give final approval of the version to be published. All authors read and approved the final manuscript.

\section{Acknowledgements}

We thank Professor F. Costantini (Department of genetics, Columbia University) and Professor Art Strauch (Department of Physiology and Cell Biology, Dorothy M. Davis Heart and Lung Research Institute, OH, USA) for providing the Cre and Smp8 containing plasmids. We thank Professor Conti, Mary Anne (NIH/NHLBI, USA) for providing SM-MHC polyclonal antibody. We also thank Professor Xiaoping Jian (Guangdong Teacher College of Foreign language and art, China) and Dr Elaina Collie-Dugui

(Department of Medicine and Therapeutics, University of Aberdeen, Scotland) for critically reading the manuscript. This research was supported by the National Natural Science Foundation of China (NO. 30230 I80). This funding covered all the cost in study design; in the collection, analysis, and interpretation of data; in the writing of the manuscript and in the decision to submit the manuscript for publication.

\section{References}

I. Kuhn C, McDonald JA: The roles of the myofibroblast in idiopathic pulmonary fibrosis. Ultrastructural and immunohistochemical features of sites of active extracellular matrix synthesis. Am J Pathol I99|, I 38(5): | 257-I265.

2. Schissel SL, Layne MD: Telomerase, myofibroblasts, and pulmonary fibrosis. Am J Respir Cell Mol Biol 2006, 34(5):520-522.

3. White ES, Atrasz RG, Hu B, Phan SH, Stambolic V, Mak TW, Hogaboam CM, Flaherty KR, Martinez FJ, Kontos CD, et al:: Negative regulation of myofibroblast differentiation by PTEN (Phosphatase and Tensin Homolog Deleted on chromosome I0). Am J Respir Crit Care Med 2006, I73(1): I I2-I2I.

4. Chambers RC, Leoni P, Kaminski N, Laurent G], Heller RA: Global expression profiling of fibroblast responses to transforming growth factor-betal reveals the induction of inhibitor of differentiation- $I$ and provides evidence of smooth muscle cell phenotypic switching. Am J Pathol 2003, 162(2):533-546.

5. Oda D, Gown AM, Vande Berg JS, Stern R: The fibroblast-like nature of myofibroblasts. Exp Mol Pathol 1988, 49(3):3।6-329.

6. Hashimoto N, Jin H, Liu T, Chensue SW, Phan SH: Bone marrowderived progenitor cells in pulmonary fibrosis. J Clin Invest 2004, I I 3(2):243-252.

7. Kasai H, Allen JT, Mason RM, Kamimura T, Zhang Z: TGF-betal induces human alveolar epithelial to mesenchymal cell transition (EMT). Respir Res 2005, 6:56.

8. Willis BC, Liebler JM, Luby-Phelps K, Nicholson AG, Crandall ED, du Bois RM, Borok Z: Induction of epithelial-mesenchymal transition in alveolar epithelial cells by transforming growth factor-betal: potential role in idiopathic pulmonary fibrosis. $\mathrm{Am}$ J Pathol 2005, 166(5): | $32 \mid-1332$.

9. Yao HW, Xie QM, Chen JQ, Deng YM, Tang HF: TGF-beta linduces alveolar epithelial to mesenchymal transition in vitro. Life Sci 2004, 76(I):29-37.

10. Kim KK, Kugler MC, Wolters PJ, Robillard L, Galvez MG, Brumwell AN, Sheppard D, Chapman HA: Alveolar epithelial cellmesenchymal transition develops in vivo during pulmonary fibrosis andis regulated by the extracellular matrix. Proc Natl Acad Sci USA 2006. 
II. Snider GL, Hayes JA, Korthy AL: Chronic interstitial pulmonary fibrosis produced in hamsters by endotracheal bleomycin: pathology and stereology. Am Rev Respir Dis 1978, I I 7(6): 1099-I I08.

12. Hinz B, Mastrangelo D, Iselin CE, Chaponnier C, Gabbiani G: Mechanical tension controls granulation tissue contractile activity and myofibroblast differentiation. Am J Pathol 200I, 159(3): 1009-1020.

13. Hamilton DL, Abremski K: Site-specific recombination by the bacteriophage PI lox-Cre system. Cre-mediated synapsis of two lox sites. J Mol Biol 1984, I 78(2):48I-486.

14. Metzger D, Clifford J, Chiba H, Chambon P: Conditional site-specific recombination in mammalian cells using a liganddependent chimeric Cre recombinase. Proc Natl Acad Sci USA 1995, 92(15):699|-6995.

15. Soriano P: Generalized lacZ expression with the ROSA26 Cre reporter strain. Nat Genet 1999, 2 I(I):70-7I.

16. Hinz B, Celetta G, Tomasek JJ, Gabbiani G, Chaponnier C: Alphasmooth muscle actin expression upregulates fibroblast contractile activity. Mol Biol Cell 200I, I 2(9):2730-274I.

17. Zhang M, Smith EP, Kuroda H, Banach W, Chernausek SD, Fagin JA: Targeted expression of a protease-resistant IGFBP-4 mutant in smooth muscle of transgenic mice results in IGFBP-4 stabilization and smooth muscle hypotrophy. J Biol Chem 2002, 277(24):21 285-2। 290.

18. Wang J, Niu W, Nikiforov $Y$, Naito S, Chernausek S, Witte D, LeRoith D, Strauch A, Fagin JA: Targeted overexpression of IGFI evokes distinct patterns of organ remodeling in smooth muscle cell tissue beds of transgenic mice. J Clin Invest 1997, I00(6): I 425-1439.

19. Zadelaar SM, Boesten LS, Pires NM, van Nieuwkoop A, Biessen EA Jukema W, Havekes LM, van Vlijmen BJ, Willems van Dijk K: Local cre-mediated gene recombination in vascular smooth muscle cells in mice. Transgenic Res 2006, I5(I):3I-36.

20. Kuhbandner S, Brummer S, Metzger D, Chambon P, Hofmann F, Feil $\mathrm{R}$ : Temporally controlled somatic mutagenesis in smooth muscle. Genesis 2000, 28(I): $15-22$

21. Zhang JC, Kim S, Helmke BP, Yu WW, Du KL, Lu MM, Strobeck M, Yu Q, Parmacek MS: Analysis of SM22alpha-deficient mice reveals unanticipated insights into smooth muscle cell differentiation and function. Mol Cell Biol 200I, 2 I (4): I336-I 344.

22. Regan CP, Manabe I, Owens GK: Development of a smooth muscle-targeted cre recombinase mouse reveals novel insights regarding smooth muscle myosin heavy chain promoter regulation. Circ Res 2000, 87(5):363-369.

23. Miano JM, Cserjesi P, Ligon KL, Periasamy M, Olson EN: Smooth muscle myosin heavy chain exclusively marks the smooth muscle lineage during mouse embryogenesis. Circ Res 1994 75(5):803-8I2.

24. Owens GK, Kumar MS, Wamhoff BR: Molecular regulation of vascular smooth muscle cell differentiation in development and disease. Physiol Rev 2004, 84(3):767-80I.

25. Grinnell $\mathrm{F}$, Ho $\mathrm{CH}$ : Transforming growth factor beta stimulates fibroblast-collagen matrix contraction by different mechanisms in mechanically loaded and unloaded matrices. Exp Cell Res 2002, 273(2):248-255.

26. Borzone G, Moreno R, Urrea R, Meneses M, Oyarzun M, Lisboa C: Bleomycin-induced chronic lung damage does not resemble human idiopathic pulmonary fibrosis. Am J Respir Crit Care Med 200I, I63(7): 1648-1653.

Publish with Bio Med Central and every scientist can read your work free of charge

"BioMed Central will be the most significant development for disseminating the results of biomedical research in our lifetime. "

Sir Paul Nurse, Cancer Research UK

Your research papers will be:

- available free of charge to the entire biomedical community

- peer reviewed and published immediately upon acceptance

- cited in PubMed and archived on PubMed Central

- yours - you keep the copyright

Submit your manuscript here:

http://www.biomedcentral.com/info/publishing_adv.asp
BioMedcentral 